\title{
AVALIAÇÃO DA EFICIÊNCIADE FUNGICIDAS NO CONTROLE DA CERCOSPORIOSE (Cercospora zeae-maydis) NA CULTURA DO MILHO ${ }^{1}$
}

\author{
NICÉSIO FILADELFO JANSSEN DE ALMEIDA PINTO², BRUNO DE ANGELIS ${ }^{3}$, MARCELO \\ HADIMU HABE ${ }^{3}$
}

\author{
${ }^{1}$ Trabalho de cooperação entre a Embrapa Milho e Sorgo e a Syngenta \\ ${ }^{2}$ Eng. Agrônomo, Pesquisador da Embrapa Milho e Sorgo. Caixa Postal 151, CEP. 35701-970 Sete Lagoas, MG. \\ nicesio@cnpms.embrapa.br (autor para correspondência). \\ ${ }^{3}$ Syngenta Proteção de Cultivos Ltda. Caixa Postal 433, CEP. 75901-970 Rio Verde, GO. E-mail: \\ bruno.angelis@syngenta.com / marcelo.habe@syngenta.com.
}

Revista Brasileira de Milho e Sorgo, v.3, n.1, p.139-145, 2004

\begin{abstract}
RESUMO - A cercosporiose do milho, causada pelo fungo Cercospora Zeae-maydis, é atualmente, no Brasil, uma das mais importantes doenças foliares dessa cultura, apresentando maior severidade de danos no sudoeste de Goiás. Este experimento foi conduzido em área de plantio direto de milho sobre milho, no município de Montividíu, GO, infectada com Cercospora zeae-maydis. O delineamento experimental foi o de blocos ao acaso, com 8 tratamentos em 4 repetições. Foi utilizado o híbrido simples Avant, de ciclo precoce. Os fungicidas foram aplicados mediante pulverizador manual pressurizado com 4 bicos pulverizadores. $O$ volume de calda aplicado em cada parcela foi de 1,4 litros. Foram utilizados os seguintes fungicidas ( $\mathrm{g}$ i.a.ha $\left.{ }^{-1}\right)$ : propiconazole $(100,0)$, propiconazole $(125,0)$, difenoconazole $(50,0)$, difenoconazole $(75,0)$, azoxystrobin $(50,0)$, azoxystrobin $(75,0)$ e tebuconazole $(200,0)$. Parcelas sem tratamento fungicida constituíram a testemunha. A primeira pulverização dos fungicidas foi realizada em plantas no estádio V8 (43 Dias Após o Plantio - DAP) e a segunda no estádio de préemborrachamento (58 DAP). Aos 85 DAP, com plantas com espigas no estádio de grão pastoso, procedeu-se à avaliação dos sintomas foliares causados por Cercospora zeae-maydis, utilizando-se do seguinte esquema de notas: $0=$ sem sintoma; 1 = poucas lesões nas folhas abaixo da espiga, nenhuma lesão acima; 2 = muitas lesões nas folhas abaixo da espiga, poucas lesões acima; 3 = severo desenvolvimento de lesões nas folhas abaixo da espiga, todas as folhas acima da espiga com lesões; 4 = todas as folhas com severo desenvolvimento de lesões, mas tecido verde ainda visível; $5=$ todas as folhas secas e mortas. Os resultados obtidos mostram que os fungicidas propiconazole, difenoconazole, azoxystrobin e tebuconazole foram eficientes no controle da cercosporiose (Cercospora zeae-maydis) do milho e todos os tratamentos fungicida garantiram a produção de grãos de milho, sendo que a maior diferença percentual foi de $38,9 \%$ em relação à testemunha. Conclui-se que os fungicidas propiconazole, difenoconazole, azoxystrobin e tebuconazole são eficientes no controle da cercosporiose do milho causada por Cercospora zeae-maydis e que a aplicação destes produtos garantem significativamente a produção de grãos.
\end{abstract}

Palavras-chave: Zea mays, fungo, produção de grãos.

\section{EVALUATION OF THE FUNGICIDE EFFICIENCY FOR THE GRAY LEAF SPOT (Cercospora zeae-maydis) CONTROL IN CORN}

ABSTRACT - The gray leaf spot of the corn, one of the most harmful foliar diseases of this crop, caused by the fungi Cercospora zeae-maydis, is now in Brazil, causing severe damage 
in Southwestern Goiás - GO. This experiment was carried out in a direct corn on corn crop area, in Montividíu, GO, infected with Cercospora zeae-maydis. The experimental design was randomized blocks, with 8 treatments in 4 replications. The simple hybrid Avant of precocious cycle was used. The fungicides were sprayed manually $\left(\mathrm{CO}_{2}\right)$ at 40 pounds of pressure using 4 pulverizing spouts. The applied volume of fungicide in each plot was of 1.4 liters. The following fungicides $\left(\mathrm{g}\right.$ a.i.ha $\left.{ }^{-1}\right)$ were used: propiconazole $(100.0)$, propiconazole (125.0), difenoconazole (50.0), difenoconazole (75.0), azoxystrobin (50.0), azoxystrobin (75.0) e tebuconazole (200.0). Plot without fungicide constituted the check. The first spray was accomplished in the V8 stage (43 days after sowing - DAS) and the second spray in the pretasseling stage (58 DAS). At 85 DAS, plants with ears in the pasty grain stage, the evaluation of the foliar symptoms of gray leaf spot was proceeded, being graded $0=$ symptomless; $1=$ little lesions below the ear, any lesion above; 2 = much lesions below the ear, little lesions above; 3 = severe development of lesions below the ear, all the leaves above the ear with lesions; $4=$ all the leaves with severe development of lesions, but green area still visible; $5=$ all the leaves dry and dead. Thus the results show that the fungicides propiconazole, difenoconazole, azoxystrobin and tebuconazole are efficient in the control of the gray leaf spot (Cercospora zeae-maydis) of the corn and that all the fungicides increased the production of corn grains, being $38.9 \%$ the largest increment.

Key words: Zea mays, fungus, grain production.

A cercosporiose do milho, causada pelo fungo Cercospora zeae-maydis, ocorreu severamente no Brasil pela primeira vez, no ano de 2000, no Sudoeste de Goiás (Rio Verde, Jataí, Montividíu, Mineiros). Atualmente, ainda é uma das mais importantes doenças foliares dessa cultura nessa região e também no Noroeste de Minas (Paracatú). Há relatos de sua ocorrência nos estados de São Paulo, Paraná, Santa Catarina, Rio Grande do Sul e Triângulo Mineiro.

A severidade da doença aumenta em condições de alta umidade relativa e temperatura diurna variando de moderada a alta, bem como em noites frias, com formação de orvalho e, principalmente, quando a temperatura se eleva logo após dias nublados ou chuvosos. Essas condições predominam nas regiões com altitudes superiores a $600 \mathrm{~m}$. Em algumas regiões tem sido observada ser mais severa nos plantios de novembro (Silva et al., 2001).

Segundo Ward et al. (1994), a cercosporiose do milho é capaz de reduzir de 20 a $60 \%$ a produção de grãos, dependendo da suscetibilidade do híbrido. Contudo, é possível promover excelente controle desse patógeno com a aplicação de fungicida.

Carlson \& Main (1976) declaram que as doenças de milho apresentam um alto grau de variabilidade espacial e temporal. Essa variabilidade causa incertezas na tomada de decisão acerca das medidas de controle, como o uso de fungicidas.

Wegulo et al. (1997) usaram um modelo para determinar os benefícios econômicos da utilização dos fungicidas chlorothalonil, propiconazole, propiconazole + chlorothalonil, mancozeb, propiconazole + mancozeb e cobre para o controle de doenças foliares em milho (Aureobasidium zeae, Bipolaris zeicola, Cercospora zeae-maydis, Exserohilum turcicum e Puccinia sorghi), e os resultados desse estudo indicaram que a utilização de fungicida na produção de sementes híbridas de milho teve resposta economicamente viável.

Por outro lado, na produção de sementes híbridas, o despendoamento mecânico das plantas progenitoras femininas remove uma quantidade significativa de área foliar e a redução adicional na área 
foliar sadia causada pela doença pode ter um efeito muito negativo sobre a produção de sementes (Bowen \& Pedersen, 1988; Wych, 1988).

Munkvold \& Martinson (1997) recomendam o controle químico da cercosporiose em milho, ressaltando que ele é economicamente viável somente em híbridos muito suscetíveis ou na produção de sementes de milho, milho pipoca e milho doce. $\mathrm{Ci}$ tam que, nos Estados Unidos, os fungicidas registrados para essa finalidade incluem o mancozeb, propiconazole, chlorothalonil, entre outros. Reportam que, para ser efetivo, um programa de aplicação de fungicida deve ser iniciado quando a doença está em níveis muito baixos ( $1 \%$ ou menos de área foliar afetada). Quando as condições são favoráveis para a doença, recomenda-se mais de uma aplicação.

De acordo com Berger (1981), a aplicação foliar de um fungicida eficiente interrompe o progresso de doença logo após a pulverização e seu efeito permanece por um "período efetivo do fungicida - PEF". O PEF é definido como o período, após a aplicação do fungicida, durante o qual há um mínimo de aumento da doença. Pulverizações com fungicidas sistêmicos apresentam PEF mais longo do que com os fungicidas protetores, o que os tornam mais eficientes no controle de doenças.

Ward et al. (1997) testaram os fungicidas benomyl (375 g i.a.ha-1 $)$ e a mistura carbendazim + flusilazole $\left(187,50+93,75\right.$ g i.a.ha $\left.{ }^{-1}\right)$ no controle da cercosporiose, sendo realizadas aplicações únicas ou múltiplas antes da antese, na antese e após a antese. Eles observaram que o controle mais efetivo da cercosporiose (Cercospora zeae-maydis) e as mais altas produções de grãos foram obtidas quando as pulverizações de fungicidas foram iniciadas no nível de severidade da doença de 2 a $3 \%$, com as aplicações em intervalos de 14 dias, continuando até a maturidade fisiológica dos grãos.

Em cultivos de produção de sementes de milho e um limitado número de cultivos comerciais de grãos nos Estados Unidos, tão bem como em cultivo de semente e milho para alimentação no Sul da África, os fungicidas são usados efetiva e economicamente no manejo de epidemias de cercosporiose. Os produtos mais efetivos foram os fungicidas sistêmicos dos grupos dos benzimidazóis e tirazóis (Carter \& Stromberg, 1992; Nowell, 1997; Stromberg, 1990; Stromberg \& Flinchum, 1993; Stromberg \& Flinchum, 1998; Ward, 1996). Mais recentemente, compostos de estrobilurinas têm também demonstrado serem efetivos (Stromberg \& Flinchum, 1993, Ward et al., 1997).

Martinson et al. (1994) estabeleceram um programa de pulverização com fungicidas para campos de produção de sementes de milho, visando o controle de diversos patógenos foliares e observaram que, quando a cercosporiose tornava-se dominante, o melhor fungicida foi o propiconazole.

No controle químico da cercosporiose (Cercospora zeae-maydis) em milho, no Brasil, Carneiro et al. (2003) reportam ganhos de rendimentos de 8,2 a 31,3\%, obtidos com aplicações dos fungicidas tebuconazole, tebuconazole + triadimenol e propiconazole + trifloxystrobin. Horst et al. (2003) relatam que azoxystrobin, propiconazole, flutriafol, trifloxystrobin + propiconazole, tebuconazole e pyraclostrobin + epoxiconazole reduziram significativamente a severidade dessa doença. Ainda segundo Fantin et al. (2003), os fungicidas carbendazim + fluquinconazole + óleo e carbendazim, seguidos por carbendazim + tebuconazole e pyraclostrobin + epoxiconazole também foram eficientes.

Adicionalmente, Juliatti et al. (2002), avaliando a eficácia de fungicidas em diferentes épocas de aplicação e híbridos, observaram que, em relação ao índice benefício, o azoxystrobin foi o mais eficiente no controle da cercosporiose. Segundo Morandi et al. (2002), em quatro e três aplicações a intervalos quinzenais, os fungicidas Priori, Palisade, Belkute, Opera e Juno reduziram significativamente 
a severidade da Cercospora zeae-maydis. Em duas e uma aplicação, apenas Opera e Priori reduziram a doença. Ottoni et al. (2002) avaliaram a eficácia dos fungicidas tebuconazole, triadimenol + tebuconazole, HEC 5725 + tebuconazole e trifloxystrobin. Os resultados mostraram correlação negativa entre severidade da doença e produção, e todos os tratamentos reduziram significativamente $o$ índice de doença. De acordo com Brandão et al. (2002), o melhor desempenho no controle dessa doença foi obtido em aplicações com azoxystrobin, propiconazole ou difenoconazole aos 45 e 60 dias após o plantio, tanto para híbridos de resistência intermediária quanto para híbridos suscetíveis.

Ramos et al. (2001) relatam que pulverização com Priori (azoxystrobin) na dose de $375 \mathrm{~mL}$ ha $^{-1}$ apresentou a menor severidade da cercosporiose e produtividade superior aos tratamentos com Cercobin (tiofanato metílico), Tilt (propiconazole), e Score (difenoconazole), com ganho de 52,33 sa$\cos /$ ha em relação à testemunha. Segundo Brandão et al. (2001), o fungicida azoxystrobin foi o mais eficiente no controle dessa doença, podendo-se adotar uma aplicação única aos 60 dias após o plantio.

O objetivo deste trabalho foi avaliar a eficiência de fungicidas no controle da cercosporiose do milho causada por Cercospora zeae-maydis.

Este experimento foi conduzido no município de Montividíu, GO, em área de plantio direto de milho sobre milho, reconhecidamente infectada com Cercospora zeae-maydis. O delineamento experimental foi o de blocos ao acaso, com 8 tratamentos em 4 repetições. Foi utilizado o híbrido simples Avant, de ciclo precoce, plantado em 31.10.2000, sendo cada parcela constituída de 8 fileiras de 10,0 $\mathrm{m}$ de comprimento e espaçadas de $0,75 \mathrm{~m}$, cuja área útil foi composta pelas 4 fileiras centrais, quantificando-se o número total de plantas nesta área colhida. Os fungicidas foram aplicados mediante a utilização de pulverizador manual pressurizado, ajustado para trabalhar com 40 libras de pressão e possuindo 4 bicos pulverizadores. $\mathrm{O}$ volume de calda fungicida aplicado em cada parcela foi de 1,4 litros.

Foram utilizados os seguintes fungicidas ( $\mathrm{g}$ i.a.ha- $\left.{ }^{-1}\right)$ : propiconazole $(100,0)$, propiconazole $(125,0)$, difenoconazole $(50,0)$, difenoconazole $(75,0)$, azoxystrobin $(50,0)$, azoxystrobin $(75,0) \mathrm{e}$ tebuconazole $(200,0)$. Parcelas sem tratamento fungicida constituíram a testemunha.

A primeira pulverização dos fungicidas foi realizada em 13.12.2000 com as plantas no estádio V8 (8 folhas completamente abertas), o qual ocorreu aos 43 dias após o plantio (DAP). A segunda aplicação foi realizada em 28.12.2000 com as plantas no estádio de pré-emborrachamento, a qual ocorreu aos 58 DAP.

Aos 85 DAP (24.01.2001), com as plantas com espigas no estádio de grão pastoso, procedeuse à avaliação da severidade dos sintomas foliares causados por Cercospora zeae-maydis, utilizandose o seguinte esquema de notas: $0=$ sem sintoma; 1 = poucas lesões nas folhas abaixo da espiga, nenhuma lesão acima; 2 = muitas lesões nas folhas abaixo da espiga, poucas lesões acima; 3 = severo desenvolvimento de lesões nas folhas abaixo da espiga, todas as folhas acima da espiga com lesões; $4=$ todas as folhas com severo desenvolvimento de lesões, mas tecido verde ainda visível; $5=$ todas as folhas secas e mortas (Hilty et al., 1979).

Os dados da produção $\left(\mathrm{kg} \mathrm{ha}^{-1}\right)$ foram corrigidos pela umidade dos grãos, ajustado a $13 \%$, em base úmida e relacionado ao número de plantas colhidas em cada parcela experimental.

Os resultados obtidos estão contidos na Tabela 1 e mostram que: 1- Os fungicidas propiconazole, difenoconazole, azoxystrobin e tebuconazole foram eficientes no controle da cercosporiose (Cercospora zeae-maydis) do milho. O controle da cercosporiose em milho obtido 
TABELA 1. Efeito do controle químico da cercosporiose (Cercospora zeae-maydis) em milho cultivar Avant, no município de Montividíu, GO. Embrapa Milho e Sorgo, Sete Lagoas, MG, 2001.

\begin{tabular}{lccc}
\hline Tratamento & Dose $\left(\mathbf{g}\right.$ i.a.ha $\left.{ }^{-1}\right)$ & $\begin{array}{c}\text { Severidade da } \\
\text { doença }\end{array}$ & $\begin{array}{c}\text { Produção de grãos } \\
\left(\mathbf{k g ~ h a}^{-\mathbf{1}}\right)\end{array}$ \\
\hline Testemunha & ------- & 3,50 & $4038,0 \mathrm{c}^{2}$ \\
Propiconazole & 100,0 & 2,37 & $5160,0 \mathrm{~b}$ \\
Propiconazole & 125,0 & 1,80 & $5610,0 \mathrm{a}$ \\
Difenoconazole & 50,0 & 2,00 & $5244,0 \mathrm{ab}$ \\
Difenoconazole & 75,0 & 2,25 & $5484,0 \mathrm{ab}$ \\
Azoxystrobin & 50,0 & 2,72 & $5418,0 \mathrm{ab}$ \\
Azoxystrobin & 75,0 & 2,50 & $5304,0 \mathrm{ab}$ \\
Tebuconazole & 200,0 & 2,00 & $5502,0 \mathrm{ab}$ \\
\hline C.V. $(\%)$ & \multicolumn{3}{c}{5,51} \\
\hline
\end{tabular}

${ }^{1}$ Escala de notas de 0 a 5 (Hilty et al., 1979)

${ }^{2} \mathrm{Na}$ coluna, as médias seguidas pelas mesmas letras não diferem entre si ao nível de $5 \%$ de significância pelo teste de Tukey.

com os fungicidas do grupo químico dos triazóis (propiconazole, difenoconazole e tebuconazole) está em conformidade com os resultados obtidos por Carter \& Stromberg (1992), Nowell (1997), Stromberg (1990), Stromberg \& Flinchum (1993) Stromberg \& Flinchum (1998) e Ward (1996). Por outro lado, o controle obtido com a estrobilurina (Priori) corrabora os relatos de Stromberg \& Flinchum, (1993), Ward et al. (1997), Juliatti et al. (2002), Morandi et al. (2002), Ramos et al. (2001) e Brandão et al. (2001). 2 - Todos os tratamentos fungicida garantiram o potencial da produção de grãos de milho, com destaque para o propiconazole a $125 \mathrm{~g}$ i.a.ha ${ }^{-1}$ que proporcionou produção superior à testemunha de $38,9 \%$. Esses resultados estão de acordo com os relatos de Ward et al. (1994), Carneiro et al. (2003) e Ramos et al. (2001). Ademais, a redução na severidade dessa doença obtida neste trabalho mediante a aplicação de fungicidas, corroboram com os resultados apresentados por Horst et al. (2003), Fantin et al. (2003), Ottoni et al. (2002) e Brandão et al. (2002).

Do exposto, conclui-se que os fungicidas propiconazole, difenoconazole, azoxystrobin e tebuconazole são eficientes no controle da cercosporiose do milho causada por Cercospora zeae-maydis e garantem significativamente a produção de grãos.

\section{Agradecimentos}

A Gilberto Rodrigues Ribeiro (Operário de campo) e a Osni Alves da Silva (Assistente de Pesquisa), pela valiosa contribuição na execução desse trabalho.

\section{Literatura Citada}

BERGER, R. D. Comparison of Gompertz and logistic equations to describe plant disease progress. Phytopathology, St. Paul, v. 71, p. 716-719, 1981.

BOWEN, K. L.; PEDERSEN, W. L. Effects of northern leaf blight and detasseling on yields and yield components of corn inbreds. Plant Disease, St. Paul, v. 72, p. 952-956, 1988.

BRANDÃO, A. M.; JULIATTI, F. C.; BRITO, C. H.; GOMES, L. S. Épocas de aplicação de fungicidas para o controle da cercosporiose (Cercospora zeaemaydis) em híbridos de milho com diferentes níveis 
de resistência. Fitopatologia Brasileira, Brasília, v. 27, p. S87, 2002. Suplemento.

BRANDÃO, A. M.; JULIATTI, F. C.; BRITO, C. H.; GOMES, L. S. Manejo integrado de cercosporiose (Cercospora zeae-maydis) em milho, no cerrado brasileiro. Fitopatologia Brasileira, Brasília, v. 26, p.401, 2001. Suplemento.

CARLSON, G. A.; MAIN, C. E. Economics of disease loss management. Annual Review of Phytopathology, Palo Alto, v. 14, p. 381-403, 1976.

CARNEIRO, L. C.; BRIGNONI, A.; PEDRIEL, F. C. Efeito de fungicidas no controle da cercosporiose do milho. Fitopatologia Brasileira, Brasília, v. 28, p. S306, 2003. Suplemento.

CARTER, M. R.; STROMBERG, E. L. The evaluation of foliar fungicides for the control of gray leaf spot disease on corn in Virginia, 1991. Fungicide and Nematicide Tests, Ithaca, v. 47, p. 169, 1992.

FANTIN, G. M.; DUARTE, A. P.; PINTO, R. A. Controle da cercosporiose do milho na safrinha. Summa Phytopathologica, Jaboticabal, v. 29, n. 1, p. 90, 2003.

HILTY, J. W.; HADDEN, C. H.; GARDEN, F. T. Response of maize hybrids and inbred lines to gray leaf spot disease and the effects on yield in Tennessee. Plant Disease Reporter, Beltsviille, v. 63, n. 6, p. 515-518, 1979.

HORST, G. C.; DUARTE, R. N.; CAMPOS, J. R.; SILVA, L. H. C. P. Eficácia do controle químico de doenças foliares em milho. Fitopatologia Brasileira, Brasília, v. 28, p. S309, 2003. Suplemento.

JULIATTI, F. C.; BRANDÃO, A. M.; BRITO, C. H.; GOMES, L. S. Manejo integrado de cercosporiose em milho e viabilidade econômica do uso de fungicidas no cerrado brasileiro.
Fitopatologia Brasileira, Brasília, v. 27, p. S121, 2002. Suplemento.

MARTINSON, C. A.; WEGULO, S. N.; RIVERA C. J. M.; NUTTER JR., F. W. Fungicidal spray program for seed corn production. In: ANNUAL CORN AND SORGHUM RESEARCH CONFERENCE, 49., 1994, Chicago. Report... Washington:ASTA, 1994. p. 71-79.

MORANDI, M. A. B.; MENEZES, C. C. E.; SANDRI, D. T.; MATOS, L. C. S. Viabilidade do uso de fungicidas no controle da cercosporiose do milho. Fitopatologia Brasileira, Brasília, v. 27, p. S138, 2002. Suplemento.

MUNKVOLD, G.; MARTINSON, C. Corn diseases. Ames: Iowa State University, 1997.25 p.

NOWEL, D. C. Studies on ear rot and gray leaf spot of maize in South Africa. 1997. Thesis (Ph.D.) - University of Natal, Pietermaritzburg.

OTTONI, G.; JULIATTI, F. C.; OLIVEIRA, W. F. de; CUNHA, M. G. da; CARVALHO, L. de S.; ALVES, W. da S.; ALBERNAZ, K. C.; ALVES, F. A. de O.; VELOSO, G. de A. Avaliação de fungicidas no controle de Cercospora zea-maydis em milho. Fitopatologia Brasileira, Brasília, v. 27, p. S145, 2002. Suplemento.

RAMOS, C. R. B. A.; MENEZES, C. C. E.; MORANDI, M. A. B. Controle químico da cercosporiose do milho. Fitopatologia Brasileira, Brasília, v. 26, p. 322, 2001. Suplemento.

SILVA, H. P.; FANTIN, G. M.; RESENDE, I. C.; PINTO, N. F. J. A.; CARVALHO, R. V. Manejo integrado de doenças na cultura do milho safrinha. In: SEMINARIO NACIONAL DE MILHO SAFRINHA, 6.; CONFERENCIA NACIONAL DE POS-COLHEITA SAG-MERCOSUL, 2.; SIMPOSIO EM ARMAZENAGEM DE GRAOS 
DO MERCOSUL, 2., 2001, Londrina. A cultura do milho safrinha: valorização da produção e conservação de grãos no Mercosul. Londrina: IAPAR, 2001. p. 113-144.

STROMBERG, E. L. The evaluation of foliar fungicides for the control of gray leaf spot in Virginia, 1989. Fungicide and Nematicide Tests, Ithaca, v. 45, p. 200, 1990.

STROMBERG, E. L.; FLINCHUM, L. E. Evaluation of foliar fungicides for the control of gray leaf spot on corn in Virginia, 1992. Fungicide and Nematicide Tests, Ithaca v. 48, p. 207, 1993.

STROMBERG, E. L.; FLINCHUM, L. E. Evaluation of foliar fungicides for the control of gray leaf spot in Virginia, 1997. Fungicide and Nematicide Tests, Ithaca, v. 53, p. 143-144, 1998.

WARD, J. M. J. Epidemiology and management of gray leaf spot: A new disease of maize in South Africa. 1996. Thesis (Ph.D.) - University of Natal, Pietermaritzburg. Falta numero de paginas e Ph.D em qual area
WARD, J. M. J.; BIRCH, E. B.; NOWELL, D. C. Grey leaf spot on maize. Pietermaritzburg: Cedara Agric. Develop. Inst., 1994. (Coordinated extension in Maize in Natal).

WARD, J. M. J.; LAING, M. D.; RIJKENBERG, F. H. J. Frequency and timing of fungicide applications for the control of gray leaf spot in maize. Plant Disease, St. Paul, v. 80, n. 1, p. 41-48, 1997.

WEGUlo, S. N.; MARTINSON, C. A.; RIVERA-C, J. M.; NUTTER JR., F. W. Model for economic analysis of fungicide usage in hybrid corn seed production. Plant Disease, St. Paul, v. 81, n. 4, p. 415-422, 1997.

WYCH, R. D. Production of hybrid seed corn. In: SPRAGUE, G. F.; DUDLEY, J. W. (Ed.) Corn and corn improvement, 3 . ed. Madison: American Society Agronomy; Crop Science Society of America; Soil Science Society of America, 1988.p. 565-607. 